TITLE:

\title{
Marine Pollution Bioassay by Sea Urchin Eggs, an Attempt to Enhance Sensitivity
}

$\operatorname{AUTHOR}(S):$

Kobayashi, Naomasa

\section{CITATION:}

Kobayashi, Naomasa. Marine Pollution Bioassay by Sea Urchin Eggs, an Attempt to Enhance Sensitivity. PUBLICATIONS OF THE SETO MARINE BIOLOGICAL LABORATORY 1990, 34(4-6): 225-237

ISSUE DATE:

1990-08-31

URL:

http://hdl.handle.net/2433/176165

RIGHT: 


\title{
Marine Pollution Bioassay by Sea Urchin Eggs, an Attempt to Enhance Sensitivity
}

\author{
By \\ Naomasa Kobayashi \\ Biological Laboratory, Doshisha University, Kamikyo-ku, Kyoto, 602, Japan
}

With Text-figure 1 and Tables $1-8$

The author (Kobayashi, 1971) proposed the use of sea urchin eggs and embryos as indicatory materials in marine pollution bioassay, and actually this was applied to the survey of the sea water pollution in the Inland Sea of Japan (Kobayashi et al., 1972). The method was then improved to enhance the sensitivity by using aged eggs (Kobayashi, 1974). Later it was found that, in successive treatment, the sensitivity to chemicals increased from fertilization to metamorphosis in the same solution, then the effects on the formation of pluteus were more pronounced than in any other earlier stages (Kobayashi, 1977). In pulse treatment, on the other hand, the sperm is most sensitive and the fertilization and gastrulation are more sensitive than the first cleavage, blastulation and pluteus formation (Kobayashi, 1980). The sperm condition was taken into consideration when an improved version of the previous method (Kobayashi, 1974) was formulated, and both aged eggs and sperm were used (Kobayashi, 1984, 1985). By using aged eggs and sperm, the sensitivity of this method has been markedly increased. Nevertheless, this improved method was too complex and a practicable method became necessary.

This paper describes another method using developing eggs and larvae up to pluteus, which is more simple and sensitive than any of the former methods. A revised ranking is also proposed to actually apply to the bioassay.

Eggs and sperm of Anthocidaris crassispina (A. Agassiz) (breeding season: May-September) or Hemicentrotus pulcherrimus (A. Agassiz) (January-March) were obtained at the Seto Marine Biological Laboratory and inseminated in each test water, then the rates of first cleavage, pluteus formation and some anomalies in the development were checked. In this method, pre-treatment of gametes, measurement of the rates of fertilization and gastrulation were excluded, differed from the former methods.

Eggs were obtained by a method $(0.5-1 \mathrm{cc}$ of $0.5 \mathrm{M}$ potassium chloride or $0.1 \mathrm{M}$ acetylchorine chloride solution injected into the body cavity), being washed several times with fresh sea water, and were used as soon as possible, within 1 hour at the latest. Sperms were obtained from testes within 6 hours after being taken out of the test. The sperm density for insemination was standardized at about 1 dry sperm: 100,000 sea water in volume. Before the beginning of experiments, the preliminary check of egg batch and sperm was made to see if the fertilization membrane was elevated in 3 minutes after insemination in over $90 \%$ of eggs and if the well synchronized first cleavage occurred in over $90 \%$ of

Publ. Seto Mar. Biol. Lab., 34(4/6), 225-237, 1990.

(Article 10) 
them in the control laboratory water. Rates of first cleavage, pluteus formation and of some abnormalities in development were checked at $26^{\circ} \mathrm{C}$ or $18^{\circ} \mathrm{C}$. In this method, the embryos or larvae deposited on the bottom were included for the check, differed from the former methods.

Series of concentrations of the chemicals to be tested were prepared by successive dilution of the

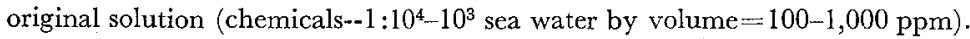

The first cleavage usually occurs about 90 minutes after the insemination at $18^{\circ} \mathrm{C}$ (warmed) for $H$. pulcherrimus or $50-60$ minutes at $26-28^{\circ} \mathrm{C}$ for A. crassispina. Hence, the rate and state of the first cleavage, namely proportions of normal two cells, undividing cells (included unfertilized eggs) and multi-cells caused by polyspermy, were checked at adequate time, respectively. Two hundred eggs were fixed with $5 \%$ formaldehyde solution at this examination. Then, the state of larvae inclusive of those deposited at the bottom, namely proportions of normal and abnormal plutei (retarded, malformed, pre-pluteus embryos and dead embryos or larvae) were checked about either 48 hours at $18^{\circ} \mathrm{C}$ or $30-36$ hours at $26-28^{\circ} \mathrm{C}$ after the insemination. In every examination, two hundred eggs, embryos or larvae were checked. These examination was repeated 3 times on different batches in respective water samples. Comparisons were made on the phases from first cleavage to pluteus between the developments in the test water of the eggs, embryos or larvae.

Indicatory developmental stages and anomalies.

The features are somewhat different between two species as shown in Figure 1.

First cleavage: Under normal conditions, the fertilized egg divides into two cells of the same size in a certain period of the time (Figs 1-A and $\mathrm{C}$ ). However, the fertilized eggs will never divide when the eggs or sperm are in bad condition or if the sea water contains toxic substances (Figs 1-B and 1-D). Polyspermic cleavage (multi-cells): The egg will divide into several cells and at the same time adopt a quite irregular configulation (Figure 1-E). Such multi-cell eggs are doomed to die sooner or later, without resuming normal development.

Gastrula: The formation of spicules and the gastral cavity begins (Figure 1-F). There are a series of successive developmental changes from blastula to gastrula, but it seems extremely difficult to use these to define exact stages. At present, only gastrulation can be used as a clearly definable criterion for the bioassay. Abnormal gastrula: Deformed (Figure 1-G) or the space-ship Apollo-like gastrula appears when the sea water contains heavy metals (Figure 1-H). Exogastrula (vegetalization): When the sea water contains toxic substances e.g. some metallic ions, the archenteron does not form inside the embryo by invagination during the gastrulation, and the tissue corresponding to the endoderm remains outside the embryo. This is the so-called "exogastrula" (Figure 1-I).

Pluteus: The ratio of normal to abnormal plutei is used as a criterion for inhibitory effect (larvae on the bottom are also considered). In normal plutei $(\mathrm{N})$ the body stage is clearly different from that of the previous stages, the digestive tract is completed and the skeleton is developed (Figs 1-J, L and N). Abnormal plutei: The formation of pluteus structures is retarded markedly (R) with their skeletal size $\leqq 1 / 2$ of normal one. Malformed plutei $\left(\mathrm{P}_{1}\right)$ appears mainly affected by gut (Fig. 1-K) or skeletal (Figs 1-M and $\mathrm{P}$ ) abnormalities. Prepluteus embryos $\left(\mathrm{P}_{2}\right)$ show exogastrulae (Fig. 1-I) or mesenchyme-filled blastulae. Dead embryos or larvae (D) also appear on the bottom. These abnormalities are found when the eggs are in bad condition or if the sea water contain lethal

Fig. 1. Normal and abnormal features in the first cleavage, gastrula and pluteus of sea urchins observed in bioassay experiments. ca. $\times 100$. A, B and N-P. Anthocidaris crassispina, test water temperature $26^{\circ} \mathrm{C}$; C-M. Hemicentrotus pulcherrimus, $18^{\circ} \mathrm{C}$. A. First cleavage (normal), 60 min. after insemination; B. Uncleaved egg (abnormal), 60 min. after; C. First cleavage (normal), $90 \mathrm{~min}$. after; D. Uncleaved egg (abnormal), $90 \mathrm{~min}$. after; E. Multi-cells (polyspermy, abnormal), 90 min. after, in Zn-seawater; F. Late gastrula (normal), 36 h. after; G. Malformed gastrula (abnormal, no skeleton), $48 \mathrm{~h}$ after, in Cd-seawater; H. Malformed gastrula (abnormal, the space-ship Apollo-like), $48 \mathrm{~h}$, in Zn-seawater; I. Malformed gastrula (abnormal, exogastrula), $48 \mathrm{~h}$, in Zn-seawater; J. Pluteus (normal, side view), $48 \mathrm{~h}$; K. Malformed pluteus (abnormal gut), $48 \mathrm{~h}$, in $\mathrm{Zn}$ seawater; L. Pluteus (normal), $48 \mathrm{~h}$; M. Malformed pluteus (abnormal skeleton), $48 \mathrm{~h}$, in Zn-seawater; N. Pluteus (normal), $48 \mathrm{~h}$; O. Retarded pluteus (abnormal, small skeleton), $48 \mathrm{~h}$, in Cd-seawater; P. Malformed pluteus (abnormal skeleton), $48 \mathrm{~h}$, in Zn-seawater. 

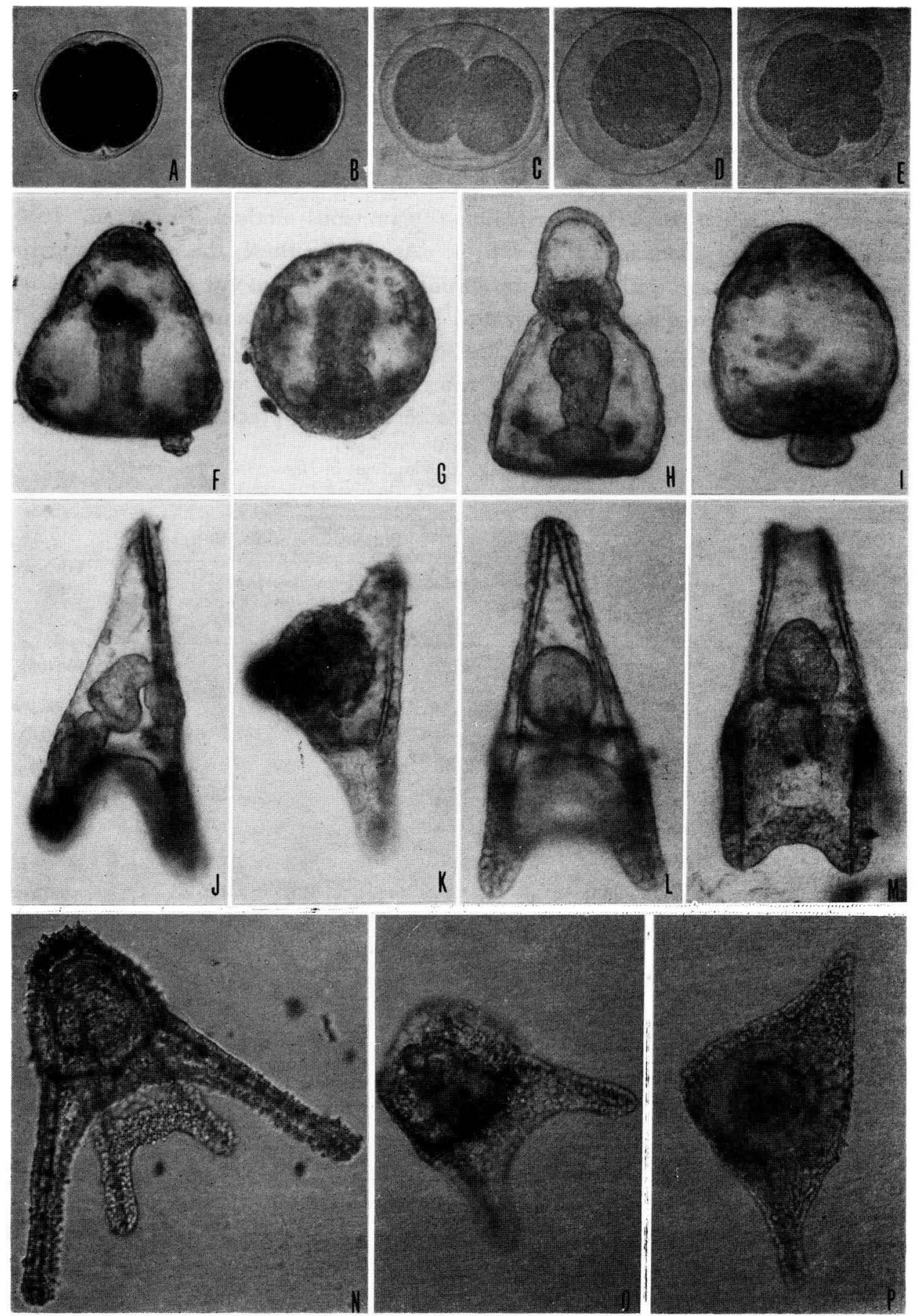

Fig. 1 
substances.

\section{Results}

Effects of some chemicals.

The bioassay results on the effects of various chemicals upon the first cleavage and pluteus formation (by new method) are compared with the developmental stages from pre-fertilization to pluteus formation (by previous method, Kobayashi, 1985) and the results are given in Tables 1 to 4 . As seen in these, the effects upon the stages at first cleavage and pluteus formation generally conform to those reported already in the previous paper (Kobayashi, 1971, 1974, 1977 and 1985). However, the procedures for the assay were decreased, i.e. the treatment of pre-fertiliation (aging of gametes) and the counting of rates of fertilization and gastrulation. On the other hand, the pluteus formation was observed more precisely. The chemicals

Table 1. Effects of various chemicals on egg development in Hemicentrotus pulcherrimus (\%). Tests Feb. 1989; water temperature $18^{\circ} \mathrm{C}$, improved method.

\begin{tabular}{|c|c|c|c|c|c|c|c|c|c|c|}
\hline \multirow[b]{2}{*}{ Chemicals } & \multirow[b]{2}{*}{$\begin{array}{l}\text { Concs. } \\
\text { (ppm) }\end{array}$} & \multicolumn{3}{|c|}{ 1st cleavage $(90 \mathrm{~min})$} & \multicolumn{5}{|c|}{ Pluteus formation (48 h) } & \multirow[b]{2}{*}{$\begin{array}{l}\text { Ultimate } \\
\text { state }\end{array}$} \\
\hline & & normal & $\begin{array}{l}1 \text { cell } \\
\text { (poly }\end{array}$ & $\begin{array}{l}\text { multi- } \\
\text { cell } \\
\text { spermy) }\end{array}$ & $\begin{array}{l}\text { normal } \\
\mathrm{N}\end{array}$ & $\begin{array}{c}\text { retard } \\
\mathrm{R}\end{array}$ & $\begin{array}{l}\text { mal- } \\
\text { form } \\
P_{1}\end{array}$ & $\begin{array}{l}\text { pre- } \\
\text { pluteus } \\
\mathrm{P}_{2}\end{array}$ & $\begin{array}{l}\text { dead } \\
\text { embryo } \\
\text { D }\end{array}$ & \\
\hline \multirow[t]{3}{*}{ Control } & & 99.0 & 1.0 & & 99.5 & 0.5 & & & & normal \\
\hline & 0.2 & 11.0 & 89.0 & & & & & & & cytolysis \\
\hline & 0.1 & 42.0 & 44.5 & 13.5 & & & & & 100 & perm. bl. \\
\hline \multirow{5}{*}{$\mathrm{CuSO}_{4} \cdot 5 \mathrm{H}_{2} \mathrm{O}$} & 0.05 & 79.5 & 14.5 & 6.0 & 28.5 & 1.0 & & 70.5 & & retard \\
\hline & 0.02 & 99.5 & 0.5 & & 37.5 & 0.5 & & 62.0 & & retard \\
\hline & 0.01 & 99.0 & 1.0 & & 93.5 & 0.5 & & 5.0 & 1.0 & normal \\
\hline & 0.2 & 30.5 & 67.5 & 2.0 & & & 34.5 & 65.5 & & perm. bl. \\
\hline & 0.1 & 75.0 & 24.5 & 5.0 & & & 51.0 & 49.0 & & exo. gast. \\
\hline \multirow[t]{5}{*}{$\mathrm{ZnCl}_{2}$} & 0.05 & 88.5 & 11.5 & & 3.0 & 0.5 & 56.5 & 40.0 & & Apollo \\
\hline & 0.02 & 92.0 & 8.0 & & 2.0 & 50.5 & 46.5 & 1.0 & & retard \\
\hline & 0.01 & 95.5 & 4.5 & & 94.5 & 0.5 & 4.5 & 0.5 & & normal \\
\hline & 5 & 41.5 & 58.5 & & & & 15.0 & 73.5 & 11.5 & perm. bl. \\
\hline & 2 & 46.0 & 52.5 & 1.5 & & & 38.5 & 55.0 & 6.5 & exo. gast. \\
\hline \multirow[t]{5}{*}{$\mathrm{NiCl}_{2} \cdot 6 \mathrm{H}_{2} \mathrm{O}$} & 1 & 48.0 & 52.0 & & & & 56.5 & 40.5 & 3.0 & Apollo \\
\hline & 0.5 & 98.5 & 1.5 & & & 80.5 & 10.5 & 6.5 & 2.5 & retard \\
\hline & 0.2 & 99.5 & 0.5 & & 94.5 & 3.0 & 0.5 & 1.5 & 0.5 & normal \\
\hline & 10 & 38.0 & 62.0 & & & & 37.0 & 61.0 & 2.0 & no skel. \\
\hline & 5 & 53.5 & 45.0 & 1.5 & & 1.0 & 56.5 & 42.0 & 0.5 & no skel. \\
\hline \multirow{3}{*}{$\underset{\mathrm{H}_{2} \mathrm{O}}{\mathrm{CdCl}_{2}} \cdot 2 \frac{1}{2}$} & 2 & 79.0 & 16.5 & 4.5 & & 97.5 & 2.5 & & & retard \\
\hline & 1 & 93.0 & 6.5 & 0.5 & 89.0 & 6.5 & 2.0 & 1.5 & 1.0 & normal? \\
\hline & 0.5 & 96.5 & 3.5 & & 95.0 & 2.5 & 2.0 & 0.5 & & normal \\
\hline
\end{tabular}

perm. bl.: permanent blastula, exo. gast.: exogastrula, Apollo: the Apollo-like gastrula, no skel.: no skeleton 
were arranged in Tables in the order of their inhibitory effects. The concentrations of chemicals used and their effects were almost the same as in the both methods.

$\mathrm{Cu}$ : In the higher concentrations of $\mathrm{Cu}$, the eggs or embryos developed in abnormal states; polyspermy induced irregular cleavage and permanent blastula occurred. Retarded development was evident in medium concentrations in most cases, but no exogastrula appeared in any cases.

$\mathrm{Zn}$ : Under the higher concentrations of $\mathrm{Zn}$, eggs and embryos developed abnormally; the elevation of many fertilization membranes was unclear or retarded and the egg maintained the appearance of an unfertilized egg until cleavage began (Table 3 showing the lower rates of fertilization than the cleavage in $\mathrm{Zn}$-water). Multi-cell cleavage induced by polyspermy (Fig. 1-E), permanent blastula and exogastrulae (Fig. 1-I) often appeared in higher concentrations, respectively. Retardation of development was evident in most cases. The space-ship Apollo-like gastrula (Fig. 1-H) also appeared in medium concentrations. Various abnormal plutei occurred with the digestive tract (Fig. 1-K) having the black mass compared

Table 2. Effects of various chemicals on egg development in Hemicentrotus pulcherrimus (\%). Tests Feb. 1989 ; water temperature $18^{\circ} \mathrm{C}$, improved method.

\begin{tabular}{|c|c|c|c|c|c|c|c|c|c|c|}
\hline \multirow[b]{2}{*}{ Chemicals } & \multirow[b]{2}{*}{$\begin{array}{c}\text { Concs. } \\
\text { ppm }\end{array}$} & \multicolumn{3}{|c|}{ Ist cleavage (90 $\mathrm{min})$} & \multicolumn{5}{|c|}{ Pluteus formation (48 h) } & \multirow[b]{2}{*}{$\begin{array}{l}\text { Ultimate } \\
\text { state }\end{array}$} \\
\hline & & normal & $\begin{array}{l}1 \text { cell } \\
\text { (pol) }\end{array}$ & $\begin{array}{l}\text { multi- } \\
\text { cell } \\
\text { yspermy) }\end{array}$ & $\begin{array}{l}\text { normal } \\
\mathrm{N}\end{array}$ & $\begin{array}{c}\text { retard } \\
\mathrm{R}\end{array}$ & $\begin{array}{c}\text { mal- } \\
\text { form } \\
\mathrm{P}_{1}\end{array}$ & $\begin{array}{l}\text { pre- } \\
\text { pluteus } \\
\mathrm{P}_{2}\end{array}$ & $\begin{array}{l}\text { dead } \\
\text { embryo } \\
\text { D }\end{array}$ & \\
\hline \multirow[t]{3}{*}{ Control } & & 98.5 & 1.5 & & 99.0 & 0.5 & & 0.5 & & normal \\
\hline & 50 & 96.5 & 3.0 & 0.5 & & 29.5 & 6.0 & 63.5 & 1.0 & no skel. \\
\hline & 20 & 98.0 & 2.0 & & 56.5 & 21.5 & 20.5 & 0.5 & 1.0 & retard \\
\hline \multirow{4}{*}{$\mathrm{MnCl}_{2} \cdot 4 \mathrm{H}_{2} \mathrm{O}$} & 10 & 97.5 & 2.0 & 0.5 & 86.5 & 6.0 & 6.5 & 0.5 & 0.5 & retard \\
\hline & 5 & 99.0 & 1.0 & & 95.5 & 2.0 & 1.5 & & 1.0 & retard \\
\hline & 2 & 98.5 & 1.5 & & 98.0 & 1.5 & 0.5 & & & normal \\
\hline & 50 & 98.0 & 2.0 & & & & & 79.5 & 20.5 & perm. bl. \\
\hline \multirow{6}{*}{$\begin{array}{l}\mathrm{Co} \\
\left(\mathrm{CH}_{3} \mathrm{COO}_{2}\right) \\
\cdot 4 \mathrm{H}_{2} \mathrm{O}\end{array}$} & 20 & 99.0 & 1.0 & & & & & 84.0 & 16.0 & no skel. \\
\hline & 10 & 97.0 & 3.0 & & 6.0 & 31.5 & 8.0 & 51.5 & 3.0 & exo. gast. \\
\hline & 5 & 98.5 & 1.5 & & 94.0 & 2.0 & 1.5 & 2.0 & 0.5 & retard \\
\hline & 2 & 99.0 & 1.0 & & 98.0 & 0.5 & 1.0 & 0.5 & & normal \\
\hline & 10 & 16.5 & 83.5 & & & & & & & cytolysis \\
\hline & 5 & 89.5 & 10.5 & & & & 1.0 & 97.5 & 1.5 & no skel. \\
\hline \multirow[t]{5}{*}{ ABS } & 2 & 86.5 & 13.5 & & 64.0 & 8.5 & 26.0 & 1.5 & & retard \\
\hline & 1 & 98.0 & 2.0 & & 91.5 & 3.5 & 4.0 & 1.0 & & retard \\
\hline & 0.5 & 97.5 & 2.5 & & 96.5 & 1.0 & 2.0 & 0.5 & & normal \\
\hline & 10 & 88.5 & 11.5 & & & & & & & cytolysis \\
\hline & 5 & 94.0 & 4.5 & 1.5 & & & & & & cytolysis \\
\hline \multirow[t]{3}{*}{$\mathrm{NH}_{4} \mathrm{Cl}$} & 2 & 97.5 & 2.5 & & & & & 100 & & perm. bl. \\
\hline & 1 & 98.5 & 1.5 & & 86.0 & 4.0 & 9.0 & 1.0 & & retard \\
\hline & 0.5 & 99.0 & 1.5 & & 98.5 & 0.5 & 1.0 & & & normal \\
\hline
\end{tabular}


with the normal (Fig. 1-J), and with the skeleton (Fig. 1-M) showing separated on the top of the larvae compared with the normal (Fig. 1-L).

$\mathrm{Ni}$ : Eggs and embryos developed more or less similar abnormality as in the case of $\mathrm{Zn}$.

$\mathrm{Cd}$ or $\mathrm{Mn}$ : In the higher concentrations of $\mathrm{Cd}$ or $\mathrm{Mn}$, the eggs or embryos developed in somewhat abnormal states as in the cases mentioned above; a few exogastrulae and the space-ship Apollo-like gastrulae appeared, however, no-skeleton or small skeleton (Figs 1-G, O) gastrulae occurred frequently. Retardation of development was evident in most cases.

Co: Eggs and embryos developed somewhat abnormally in higher concentrations of Co; permanent blastula, no-skeleton gastrula and exogastrula also appeared.

Table 3. Effects of various chemicals on egg development in Hemicentrotus pulcherrimus. Test Feb. 1989; Water temperature $18^{\circ} \mathrm{C}$. Pre-treated eggs $(3 \mathrm{~h})$-pre-treated sperms $(5 \mathrm{~min})$. 1985 method.

\begin{tabular}{|c|c|c|c|c|c|c|c|c|c|c|c|}
\hline \multirow[b]{3}{*}{ Chemicals } & \multirow[b]{3}{*}{$\begin{array}{l}\text { Concs. } \\
\text { (ppm) }\end{array}$} & \multicolumn{9}{|c|}{ Time after insemination } & \multirow[t]{3}{*}{ Ultimate state } \\
\hline & & \multirow{2}{*}{$\frac{3 \min }{20}$} & \multicolumn{3}{|c|}{$90 \mathrm{~min}$} & \multicolumn{3}{|c|}{$24 \mathrm{~h}$} & \multicolumn{2}{|c|}{$48 \mathrm{~h}$} & \\
\hline & & & 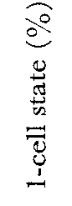 & 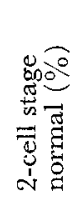 & 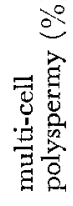 & 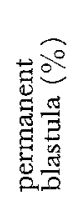 & 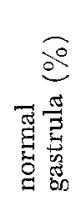 & $\frac{2}{2}$ & 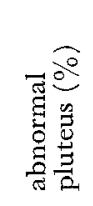 & 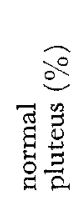 & \\
\hline \multirow[t]{3}{*}{ Control } & & 99.0 & 1.0 & 99.0 & 0 & 0.5 & 99.5 & 0 & 0.5 & 99.5 & normal \\
\hline & 0.2 & 3.0 & 96.5 & 3.5 & 0 & & & & & & cytolysis \\
\hline & 0.1 & 50.5 & 53.0 & 34.5 & 12.5 & 81.5 & 18.5 & 0 & & & permanent blastula \\
\hline \multirow{5}{*}{$\mathrm{CuSO}_{4} \cdot 5 \mathrm{H}_{2} \mathrm{O}$} & 0.05 & 82.5 & 16.5 & 78.0 & 5.5 & 29.0 & 71.0 & 0 & 81.5 & 18.5 & retardation \\
\hline & 0.02 & 93.5 & 9.5 & 90.5 & 0 & 10.5 & 89.5 & 0 & 73.5 & 26.5 & retardation \\
\hline & 0.01 & 98.0 & 4.5 & 95.5 & 0 & 3.0 & 97.0 & 0 & 4.0 & 96.0 & normal \\
\hline & 0.2 & 5.5 & 77.0 & 23.0 & 0 & 72.5 & 17.5 & 10.0 & & & permanent blastula \\
\hline & 0.1 & 40.0 & 30.0 & 65.5 & 4.5 & 63.5 & 13.0 & 23.5 & & & permanent blastula \\
\hline \multirow[t]{5}{*}{$\mathrm{ZnCl}_{2}$} & 0.05 & 78.5 & 27.0 & 73.0 & 0 & 21.5 & 46.0 & 32.5 & 100 & 0 & retardation \\
\hline & 0.02 & 94.5 & 9.0 & 91.0 & 0 & 3.0 & 91.5 & 5.5 & 75.5 & 24.5 & retardation \\
\hline & 0.01 & 97.0 & 4.0 & 96.0 & 0 & 2.0 & 98.0 & 0 & 6.0 & 94.0 & normal \\
\hline & 5 & 38.5 & 63.0 & 37.0 & 0 & 87.0 & 11.5 & 1.5 & & & permanent blastula \\
\hline & 2 & 48.5 & 51.0 & 47.0 & 2.0 & 41.5 & 23.5 & 35.0 & 89.5 & 10.5 & exogastrula \\
\hline \multirow[t]{5}{*}{$\mathrm{NiCl}_{2} \cdot 6 \mathrm{H}_{2} \mathrm{O}$} & 1 & 51.5 & 49.5 & 50.5 & 0 & 23.0 & 55.0 & 22.0 & 86.5 & 13.5 & retardation \\
\hline & 0.5 & 82.0 & 18.5 & 81.5 & 0 & 11.5 & 82.5 & 6.0 & 68.0 & 32.0 & retardation \\
\hline & 0.2 & 97.5 & 3.5 & 96.5 & 0 & 3.0 & 97.0 & 0 & 3.5 & 96.5 & normal \\
\hline & 10 & 20.5 & 80.0 & 18.5 & 1.5 & 98.0 & 2.0 & 0 & & & permanent blastula \\
\hline & 5 & 72.5 & 24.5 & 68.5 & 7.0 & 31.5 & 68.5 & 0 & 28.5 & 71.5 & retardation \\
\hline \multirow{3}{*}{$\mathrm{CdCl}_{2} \cdot 2 \cdot \frac{1}{2} \mathrm{H}_{2} \mathrm{O}$} & 2 & 81.5 & 17.0 & 80.0 & 3.0 & 24.5 & 73.5 & 2.0 & 18.0 & 82.0 & retardation \\
\hline & 1 & 92.0 & 9.0 & 91.0 & 0 & 8.0 & 92.0 & 0 & 7.5 & 92.5 & normal? \\
\hline & 0.5 & 96.0 & 4.5 & 95.5 & 0 & 3.0 & 97.0 & 0 & 4.5 & 95.5 & normal \\
\hline
\end{tabular}


Table 4. Effects of various chemicals on egg development in Hemicentrotus pulcherrimus. Test Feb. 1989; Water temperature $18^{\circ} \mathrm{C}$. Pre-treated eggs $(3 \mathrm{~h})+$ pre-treated sperms $(5 \mathrm{~min})$. 1985 method.

\begin{tabular}{|c|c|c|c|c|c|c|c|c|c|c|c|}
\hline \multirow[b]{3}{*}{ Chemicals } & \multirow[b]{3}{*}{$\begin{array}{l}\text { Concs. } \\
\text { (ppm) }\end{array}$} & \multicolumn{9}{|c|}{ Time after insemination } & \multirow[t]{3}{*}{ Ultimate state } \\
\hline & & \multirow{2}{*}{$\frac{3 \min }{\widehat{2}}$} & \multicolumn{3}{|c|}{$90 \mathrm{~min}$} & \multicolumn{3}{|c|}{$24 \mathrm{~h}$} & \multicolumn{2}{|c|}{$48 \mathrm{~h}$} & \\
\hline & & & 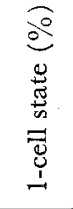 & 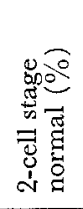 & 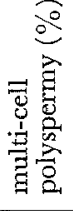 & 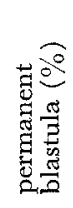 & 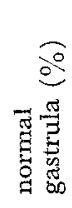 & $\frac{2}{2}$ & 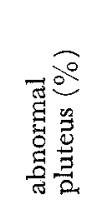 & 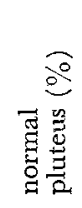 & \\
\hline \multirow[t]{3}{*}{ Control } & & 99.5 & 1.0 & 99.0 & 0 & 1.0 & 99.0 & 0 & 0.5 & 99.5 & normal \\
\hline & 50 & 70.5 & 34.5 & 65.0 & 0.5 & 83.5 & 15.0 & 1.5 & & & permanent blastula \\
\hline & 20 & 90.5 & 11.5 & 88.5 & 0 & 53.5 & 42.0 & 4.5 & 67.5 & 32.5 & retardation \\
\hline \multirow[t]{5}{*}{$\mathrm{MnCl}_{2} \cdot 4 \mathrm{H}_{2} \mathrm{O}$} & 10 & 97.0 & 5.0 & 95.0 & 0 & 11.5 & 88.5 & 0 & 21.5 & 78.5 & retardation \\
\hline & 5 & 98.5 & 3.0 & 97.0 & 0 & 3.5 & 96.5 & 0 & 7.5 & 92.5 & retardation \\
\hline & 2 & 99.0 & 1.5 & 98.5 & 0 & 3.0 & 97.0 & 0 & 2.0 & 98.0 & normal \\
\hline & 50 & 74.5 & 26.5 & 73.5 & 0 & 100 & 0 & 0 & & & permanent blastula \\
\hline & 20 & 85.5 & 15.5 & 83.0 & 1.5 & 21.0 & 64.5 & 14.5 & 100 & 0 & retardation \\
\hline \multirow{5}{*}{$\begin{array}{c}\mathrm{Co}\left(\mathrm{CH}_{3} \mathrm{COO}\right)_{2} \\
\cdot 4 \mathrm{H}_{2} \mathrm{O}\end{array}$} & 10 & 94.5 & 6.0 & 93.5 & 0.5 & 9.5 & 88.0 & 2.5 & 97.5 & 2.5 & retardation \\
\hline & 5 & 98.0 & 2.5 & 97.5 & 0 & 4.5 & 93.5 & 2.0 & 8.5 & 91.5 & retardation \\
\hline & 2 & 99.0 & 1.5 & 98.5 & 0 & 1.5 & 98.5 & 0 & 2.0 & 98.0 & normal \\
\hline & 5 & 21.5 & 82.0 & 18.0 & 0 & & & & & & cytolysis \\
\hline & 2 & 72.0 & 29.5 & 70.5 & 0 & 40.5 & 59.5 & 0 & 100 & 0 & no skeleton \\
\hline \multirow[t]{5}{*}{ ABS } & 1 & 88.5 & 13.0 & 87.0 & 0 & 21.0 & 79.0 & 0 & 83.5 & 16.5 & no skeleton \\
\hline & 0.5 & 93.5 & 7.0 & 93.0 & 0 & 9.5 & 90.5 & 0 & 51.0 & 49.0 & retardation \\
\hline & 0.2 & 98.5 & 1.5 & 98.5 & 0 & 2.0 & 98.0 & 0 & 3.5 & 96.5 & normal \\
\hline & 10 & 80.5 & 21.5 & 78.5 & 0 & & & & & & cytolysis \\
\hline & 5 & 93.5 & 7.0 & 92.0 & 1.0 & 100 & 0 & 0 & & & permanent blastula \\
\hline \multirow[t]{3}{*}{$\mathrm{NH}_{4} \mathrm{Cl}$} & 2 & 96.5 & 4.0 & 93.5 & 2.5 & 63.5 & 36.5 & 0 & 100 & 0 & permanent blastula \\
\hline & 1 & 98.5 & 2.0 & 98.0 & 0 & 13.5 & 86.5 & 0 & 12.5 & 87.5 & retardation \\
\hline & 0.5 & 99.0 & 2.0 & 98.0 & 0 & 3.0 & 97.0 & 0 & 2.5 & 97.5 & normal \\
\hline
\end{tabular}

Retardation of development was evident in most cases.

ABS: Higher concentrations of ABS reduced the development or caused by cytolysis of cells. There was no evidence of polyspermy. The development was anomalous (no-skeleton) or retarded even in lower concentrations. No exogastrula and other abnormal gastrulae appeared in any cases.

$\mathrm{NH}_{3}$ : Higher concentrations of $\mathrm{NH}_{3}$ induced the cytolysis after the cleavage. polyspermy occurred in higher concentrations. Exogastrula and other abnormal gastrulae did not appear in any cases. Permanent blastula and retardation of the development were evident in most cases.

Effects of marine pollution.

The marine pollution boassay was carried out concerning with the first cleavage 
and the pluteus formation (new method) compared with the previous method (Kobayashi, 1985), as in the above mentioned experiments with some chemicals, on the water samples collected at the four stations around Hatakejima. Island (Tables 5, 6).

The effects upon the first cleavage and pluteus formation of the fertilized eggsand the fertilization, first cleavage, gastrulation and pluteus formation of the fertilized eggs by aged gametes as the previous method-in the polluted sea water were examined by comparing one another. The figures concerning these developmental processes, obtained in respective test water samples and those observed in the control water, the running sea water of the laboratory were checked. The rates of normal pluteus were shown more or less lower by the new method than the previous one at the more polluted locations. It was found, then, that the effects become clearer with the pluteus formation on one side and somewhat pronounced with the degree of pollution ( 0 to 1 ) of the water on the other side, as compared with Tables 5 and 6 at the bottom water from land side of Hatakejima. Actually, the effect was some-

Table 5. Results of the July 19, 1988 experiment with eggs of Anthocidaris crassispina (\%). Test water temperature; $26^{\circ} \mathrm{C}$. *After improved ranking (Ranking IV)

\begin{tabular}{|c|c|c|c|c|c|c|c|c|c|}
\hline \multirow[b]{2}{*}{$\begin{array}{l}\text { Location } \\
\text { (depth) }\end{array}$} & \multicolumn{3}{|c|}{ 1st cleavage $(60 \mathrm{~min})$} & \multicolumn{5}{|c|}{ Pluteus formation $(36 \mathrm{~h}$ ) } & \multirow{2}{*}{$\begin{array}{l}\text { *Degree } \\
\text { of } \\
\text { inhibi- } \\
\text { tory } \\
\text { effect }\end{array}$} \\
\hline & normal & $\begin{array}{l}1 \text { cell } \\
\text { (polyst }\end{array}$ & $\begin{array}{l}\text { multi- } \\
\text { cell } \\
\text { permy) }\end{array}$ & $\begin{array}{l}\text { normal } \\
\mathrm{N}\end{array}$ & $\begin{array}{c}\text { retard } \\
\quad \mathrm{R}\end{array}$ & $\begin{array}{l}\text { mal- } \\
\text { form } \\
P_{1}\end{array}$ & $\begin{array}{l}\text { pre- } \\
\text { pluteus } \\
\mathbf{P}_{2}\end{array}$ & $\begin{array}{l}\text { dead } \\
\text { embryo } \\
\text { D }\end{array}$ & \\
\hline \multirow{3}{*}{$\begin{array}{l}\text { Running } \\
\text { sea water of } \\
\text { Laboratory }\end{array}$} & 98.0 & 2.0 & 0 & 99.0 & 1.0 & 0 & 0 & 0 & \multirow{3}{*}{0} \\
\hline & 99.0 & 0.5 & 0.5 & 99.5 & 0.5 & 0 & 0 & 0 & \\
\hline & 99.5 & 0.5 & 0 & 99.0 & 0 & 1.0 & 0 & 0 & \\
\hline \multirow{4}{*}{$\begin{array}{l}\text { Water from } \\
\text { open sea side of } \\
\text { Hatakejima } \\
\text { Surface }\end{array}$} & 98.5 & 1.5 & 0 & 96.0 & 0 & 2.0 & 2.0 & 0 & \multirow{3}{*}{0} \\
\hline & 98.5 & 0.5 & 1.0 & 98.5 & 0 & 1.5 & 0 & 0 & \\
\hline & 99.0 & 1.0 & 0 & 99.5 & 0 & 0.5 & 0 & 0 & \\
\hline & 96.5 & 3.5 & 0 & 96.5 & 0.5 & 3.0 & 0 & 0 & \multirow{3}{*}{0} \\
\hline \multirow[t]{2}{*}{ Bottom $(15 \mathrm{~m})$} & 97.0 & 2.0 & 1.0 & 96.5 & 1.5 & 1.5 & 0.5 & 0 & \\
\hline & 98.5 & 1.5 & 0 & 98.0 & 1.0 & 1.0 & 0 & 0 & \\
\hline \multirow{3}{*}{$\begin{array}{l}\text { Water from land } \\
\text { side of Hatakejima } \\
\text { Surface }\end{array}$} & 98.0 & 2.0 & 0 & 86.5 & 4.0 & 9.5 & 0 & 0 & \multirow{3}{*}{1} \\
\hline & 98.5 & 1.5 & 0 & 93.0 & 1.0 & 6.0 & 0 & 0 & \\
\hline & 98.0 & 2.0 & 0 & 91.0 & 1.5 & 7.5 & 0 & 0 & \\
\hline \multirow{3}{*}{ Bottom $(7 \mathrm{~m})$} & 99.0 & 1.0 & 0 & 96.5 & 0 & 3.0 & 0.5 & 0 & \multirow{3}{*}{1} \\
\hline & 98.0 & 1.5 & 0.5 & 95.0 & 0.5 & 4.0 & 0.5 & 0 & \\
\hline & 99.0 & 1.0 & 0 & 95.5 & 4.0 & 0.5 & 0 & 0 & \\
\hline \multirow{3}{*}{$\begin{array}{l}\text { Sea water from } \\
\text { Tsunashirazu cove } \\
\text { Surface }\end{array}$} & 98.0 & 2.0 & 0 & 90.5 & 1.0 & 4.5 & 4.0 & 0 & \multirow{3}{*}{1} \\
\hline & 96.5 & 3.0 & 0.5 & 88.5 & 4.5 & 3.5 & 3.5 & 0 & \\
\hline & 98.0 & 2.0 & 0 & 90.0 & 2.0 & 4.0 & 4.0 & 0 & \\
\hline \multirow{3}{*}{ Bottom (5 m) } & 98.0 & 2.0 & 0 & 90.0 & 4.0 & 3.0 & 3.0 & 0 & \multirow{3}{*}{1} \\
\hline & 98.0 & 2.0 & 0 & 89.0 & 4.0 & 3.5 & 3.5 & 0 & \\
\hline & 96.0 & 3.5 & 0.5 & 90.0 & 3.5 & 4.5 & 2.0 & 0 & \\
\hline
\end{tabular}


Table 6. Results of the July 19, 1988 experiment with eggs of Anthocidaris crassispina. Wind 0; Test water temperature $26^{\circ} \mathrm{C}$; 5 mins old sperms, $3 \mathrm{hrs}$ old eggs. *After ranking III 1985

\begin{tabular}{|c|c|c|c|c|c|c|c|c|c|c|}
\hline \multirow{3}{*}{$\begin{array}{l}\text { Location } \\
\text { (depth) }\end{array}$} & \multicolumn{9}{|c|}{ Time after insemination } & \multirow{3}{*}{$\begin{array}{l}* \text { Degree of } \\
\text { inhibitory } \\
\text { effect }\end{array}$} \\
\hline & \multirow{2}{*}{$\frac{3 \mathrm{~min}}{20}$} & \multicolumn{3}{|c|}{$60 \mathrm{~min}$} & \multicolumn{3}{|c|}{$18 \mathrm{~h}$} & \multicolumn{2}{|c|}{$36 \mathrm{~h}$} & \\
\hline & & 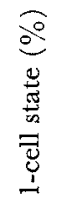 & 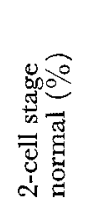 & 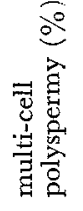 & 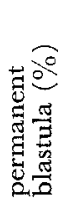 & 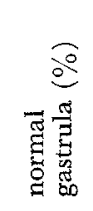 & 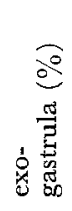 & 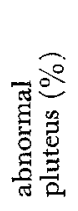 & 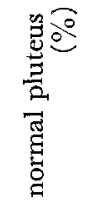 & \\
\hline \multirow{3}{*}{$\begin{array}{l}\text { Running } \\
\text { sea water of } \\
\text { Laboratory }\end{array}$} & 97.0 & 3.5 & 96.5 & 0 & 0.5 & 99.5 & 0 & 0 & 100 & \\
\hline & 99.5 & 1.0 & 99.0 & 0 & 0.5 & 99.5 & 0 & 0.5 & 99.5 & 0 \\
\hline & 99.0 & 1.5 & 98.5 & 0 & 0 & 100 & 0 & 0.5 & 99.5 & \\
\hline \multirow{4}{*}{$\begin{array}{l}\text { Water from open } \\
\text { sea side of } \\
\text { Hatakejima } \\
\text { Surface }\end{array}$} & 98.5 & 2.0 & 98.0 & 0 & 0 & 100 & 0 & 0.5 & 99.5 & \\
\hline & 98.5 & 1.5 & 98.5 & 0 & 0.5 & 99.5 & 0 & 0.5 & 99.5 & 0 \\
\hline & 98.5 & 1.5 & 98.5 & 0 & 0.5 & 99.5 & 0 & 0.5 & 99.5 & \\
\hline & 96.5 & 4.0 & 96.0 & 0 & 1.5 & 98.5 & 0 & 1.0 & 99.0 & \\
\hline \multirow[t]{2}{*}{ Bottom (15 m) } & 98.0 & 2.0 & 98.0 & 0 & 1.0 & 99.0 & 0 & 1.5 & 98.5 & 0 \\
\hline & 98.0 & 2.5 & 97.5 & 0 & 1.0 & 99.0 & 0 & 2.0 & 98.0 & \\
\hline \multirow{4}{*}{$\begin{array}{l}\text { Water from } \\
\text { land side of } \\
\text { Hatakejima } \\
\text { Surface }\end{array}$} & 98.5 & 2.0 & 98.0 & 0 & 4.5 & 95.5 & 0 & 7.0 & 93.0 & \\
\hline & 99.0 & 1.5 & 98.5 & 0 & 8.5 & 91.5 & 0 & 10.5 & 89.5 & 1 \\
\hline & 98.0 & 2.5 & 97.5 & 0 & 7.0 & 93.0 & 0 & 9.5 & 90.5 & \\
\hline & 98.0 & 2.5 & 97.0 & 0.5 & 3.0 & 97.0 & 0 & 2.5 & 97.5 & \\
\hline \multirow[t]{2}{*}{ Bottom (7 m) } & 98.0 & 2.0 & 98.0 & 0 & 3.5 & 96.5 & 0 & 3.0 & 97.0 & 0 \\
\hline & 98.5 & 2.5 & 97.5 & 0 & 2.5 & 97.5 & 0 & 3.0 & 97.0 & \\
\hline \multirow{4}{*}{$\begin{array}{l}\text { Sea water from } \\
\text { Tsunashirazu } \\
\text { cove } \\
\text { Surface }\end{array}$} & 95.0 & 8.5 & 91.5 & 0 & 2.5 & 97.5 & 0 & 3.0 & 97.0 & \\
\hline & 98.5 & 3.0 & 97.0 & 0 & 5.0 & 95.0 & 0 & 4.5 & 95.5 & 1 \\
\hline & 97.5 & 3.5 & 96.5 & 0 & 4.0 & 96.0 & 0 & 4.0 & 96.0 & \\
\hline & 98.0 & 2.0 & 98.0 & 0 & 3.5 & 96.5 & 0 & 3.5 & 96.5 & \\
\hline \multirow[t]{2}{*}{ Bottom (5 m) } & 98.0 & 2.5 & 97.5 & 0 & 4.0 & 96.0 & 0 & 3.0 & 97.0 & 1 \\
\hline & 95.0 & 6.5 & 93.5 & 0 & 5.5 & 94.5 & 0 & 4.0 & 96.0 & \\
\hline
\end{tabular}

what remarkable in the water samples from the land side of Hatakejima where the water was polluted heavily by waste products of fish rearing, etc.

\section{Considerations and Proposal of a New Ranking for Bioassay}

Actually it was reported by Kobayashi (1977), though the analysis was dealt only roughly, that such effect on pluteus formation was much more pronounced when eggs and larvae were reared successively in the same water. In this method, measurement of the rates of fertilization and gastrulation were excluded for save time. As described before, under the higher concentrations of Zn, the elevation of 
many fertilization membranes was unclear or retarded and the egg maintained the appearance of an unfertilized egg until cleavage began. Furthermore, some species of the urchins, i.e. Anthocidaris crassispina, the elevation of fertilization membrane was somewhat difficult to observe (Fig. 1-B). As the results of like these, counting of the rates of fertilization were omitted. Moreover, the measurement of the rates of gastrulation was omitted because pluteus formation was observed precisely.

On the pluteus formation, the teratogenic effects of some chemicals were studied by Hagström \& Lönning (1973) and Pagano, Esposito \& Giordano (1982). Recently, Pagano et al. (1986) were worked these more precisely on pluteus formation. This method was adopted for the present works. In normal pluteus (N), the digestive tract and skeleton are developed well. The structure of skeleton at the top of pluteus are different with species. The skeletal structure of Hemicentrotus pulcherrimus is simple, two parts of the skeleton fuse at the top (Fig. 1-L). In contrast, the structure of Anthocidaris crassispina is complex, making the basket-like near the top (Fig. $1-\mathrm{N}$ ). So, it is easy to observe the abnormal structure in H. pulcherrimus (Fig. 1-M, separated at the top). In abnormal plutei, the retarded (R) formation of pluteus structure were shown very often in every cases. Malformed plutei $\left(P_{1}\right)$ appeared mainly affected by gut and skeleton, especially in the latter in pollutant. In prepluteus embryos $\left(\mathrm{P}_{2}\right)$ : the space ship Apollo-like gastrula and exogastrula were shown treated with metals especially in $\mathrm{Zn}, \mathrm{Ni}$ and $\mathrm{Co}$; no-skeleton gastrula appeared in $\mathrm{Cd}, \mathrm{Mn}$, Co and ABS; and mesenchyme-filled blastula or permanent blastula were found frequently in all cases. Dead embryos or larvae (D) appeared as the partial or whole cytolysis on the bottom. As described above and Tables 1-4 indicated that the present new method was shown nearly the same sensitivity, save the time, more simple and multi-parametric than the previous one.

On the marine pollution boassay, the effects upon the first cleavage and pluteus formation of the fertilized eggs (new method) and the fertilization, first cleavage, gastrulation and pluteus formation of the fertilized eggs by aged gametes in the polluted water (previous method) were examined by comparing one another. The rates of normal pluteus were shown more or less lower by the new method than the previous one at the more polluted locations. It was found, however, that the effects become clearer with the pluteus formation on one side and somewhat pronounced with the degree of pollution of the water $(0$ to 1$)$ on the other side. As seen in these experiments, in marine pollution bioassay using sea urchin eggs, it will be better to check first cleavage and pluteus formation precisely.

Thus, it is apparent that the method of grading the pollution degree proposed by Kobayashi (1985, the Ranking III, Table 7) has been greatly improved by checking the developmental stages in first cleavage and pluteus formation. On the basis of these experiments, the new Ranking IV is proposed here (Table 8). In this method, pre-treatment of gametes (aging) and measurement of the rates of fertilization and gastrulation were excluded. However, the formations of normal and abnormal plutei were checked precisely. Furthermore, inhibitory degrees (Grades) were decreased from 6 to 4 . In general, the Ranking IV is sensitive, save the time 
Table 7. An improved ranking (the Ranking III) of the sea water pollution by using aged eggs and aged sperms of sea urchins.

\begin{tabular}{|c|c|c|c|c|c|c|c|}
\hline \multirow{2}{*}{ Inhibitory degree } & \multirow{2}{*}{\multicolumn{2}{|c|}{$\begin{array}{l}\text { Stage } \\
\text { Grade }\end{array}$}} & \multirow{2}{*}{\multicolumn{2}{|c|}{$\begin{array}{l}\text { Fertiliz. } \\
\begin{array}{l}\text { membrane } \\
\text { formation }\end{array}\end{array}$}} & \multicolumn{3}{|c|}{ First cleavage } \\
\hline & & & & & 1 cell & $\begin{array}{c}2 \text { cell } \\
\text { (normal) }\end{array}$ & $\begin{array}{l}\text { multi-cells* } \\
\text { (polyspermy) }\end{array}$ \\
\hline \multicolumn{2}{|l|}{ Violent inhibition } & 5 & \multicolumn{2}{|c|}{$0-50 \%$} & $100-50 \%$ & $0-50 \%$ & $15-100 \%$ \\
\hline \multicolumn{2}{|l|}{ Strong inhibition } & 4 & \multicolumn{2}{|c|}{$51-60$} & $49-40$ & $51-60$ & $12-14$ \\
\hline \multicolumn{2}{|l|}{ Moderate inhibition } & 3 & \multicolumn{2}{|c|}{$61-70$} & $39-30$ & $61-70$ & $9-11$ \\
\hline \multicolumn{2}{|l|}{ Weak inhibition } & 2 & \multicolumn{2}{|c|}{$71-80$} & $29-20$ & $71-80$ & $6-\quad 8$ \\
\hline Slight inhibition & & 1 & \multicolumn{2}{|c|}{$81-90$} & $19-10$ & $81-90$ & $3-5$ \\
\hline No inhibition & & 0 & \multicolumn{2}{|c|}{$91-100$} & $9-0$ & $91-100$ & $0-2$ \\
\hline \multirow{2}{*}{ Inhibitory degree } & \multicolumn{4}{|c|}{ Gastrulation } & \multicolumn{2}{|c|}{ Pluteus formation } & \multirow{2}{*}{ Remarks**: } \\
\hline & $\begin{array}{c}\text { permanent } \\
\text { blastula }\end{array}$ & $\begin{array}{l}\text { gast } \\
\text { (nor }\end{array}$ & & cxogastrula* & abnormal & normal & \\
\hline Violent inhibition & $100-25 \%$ & & $5 \%$ & $15-100 \%$ & $100-25 \%$ & $0-75 \%$ & $\begin{array}{l}\text { development stop- } \\
\text { ped in early stages }\end{array}$ \\
\hline Strong inhibition & $24-20$ & $76-$ & & $12-14$ & $24-20$ & $76-80$ & $\begin{array}{l}\text { development de- } \\
\text { layed or deformed }\end{array}$ \\
\hline Moderate inhibition & $19-15$ & $81-$ & & 9- 11 & $19-15$ & $81-85$ & $\begin{array}{l}\text { development some- } \\
\text { what delayed and } \\
\text { deformed }\end{array}$ \\
\hline Weak inhibition & $14-10$ & $86-$ & & $6-8$ & $14-10$ & $86-90$ & \\
\hline Slight inhibition & $9-5$ & $91-$ & & $3-5$ & $9-5$ & $91-95$ & \\
\hline No inhibition & $4-0$ & $96-$ & & $0-2$ & $4-0$ & $96-100$ & \\
\hline
\end{tabular}

*: Rather infrequent. **: Notes when such features were seen on over $50 \%$ of the checked embryos. Hours to insemination are 3 hours in summer (water temperature $26-28^{\circ} \mathrm{G}$ ) for Anthocidaris eggs, 9 hours in autumn (water temperature $13-16^{\circ} \mathrm{G}$ ) for Pseudocentrotus eggs and 6 hours in winter (water temperature $17-19^{\circ} \mathrm{C}$, warmed) for Hemicentrotus eggs (after Kobayashi, 1985).

Table 8. Ranking of the sea water pollution by using sea urchin eggs (Ranking IV)

\begin{tabular}{lcccc}
\hline Inhibitory degree & Grade & First cleavage & $\begin{array}{c}\text { Pluteus formation } \\
\text { (abnormal }\end{array}$ \\
\hline Strong inhibition & 3 & $50-100 \%$ & $15-100 \%$ & $50-100 \%$ \\
Moderate inhibition & 2 & $30-49$ & $9-14$ & $30-49$ \\
Weak inhibition & 1 & $10-29$ & $3-8$ & $5-29$ \\
No inhibition & 0 & $0-9$ & $0-2$ & $0-4$ \\
\hline
\end{tabular}

* Retarded, malformed, pre-pluteus embryos and dead embryos or larvae

and more simple one than the Ranking III. Therefore, the Rankings III and I (Kobayashi, 1971) will be no longer used by the present author. From these point of view, the present method will be valuable for the marine pollution bioassay. 


\section{Summary}

To improve the previous methods of marine pollution bioassay presented by the author (Kobayashi, 1971, 1974, 1977 and 1985), especially to enhance the sensitivity, some experiments were made using two species of sea urchins, Anthocidaris crassispina and Hemicentrotus pulcherrimus. The effects of various chemicals and waters upon the eggs and embryos in respective developmental stages at first cleavage and pluteus were observed. Rates of first cleavage, pluteus stages and some anomalies of development in the test water were checked. Various anomalities, i.e. the absence of cleavage, irregular cleavage induced by polyspermy, abnormal development and cytolysis, etc., occurred sensitively in the series of experiments. The irregularity was most abundantly seen at the stage of pluteus, i.e. retarded, malformed, pre-pluteus embryos and dead embryos or larvae. This result is available for improving the bioassay of marine pollution. On the basis of these experiments, a new ranking of the sea water pollution (Ranking IV) is proposed. It is sensitive, saves the time and more simple method than the Ranking III and I (1985 and 1971).

\section{Acknowledgements}

A part of this work was desinged at the Stazione Zoologica di Napoli in spring of 1988, as a Scientific Collaboration in Naples aided by the National Research Council of Italy and the Japan Society for the Promotion of Science. The author wishes to express his hearty thanks to the director and the staff of the Stazione Zoologica di Napoli for facilities given to him, and to Dr. G. Pagano of the Istituto Nazionale Tumori for advice. The author acknowledges with greatful thanks the assistance provided by Prof. E. Harada, Dr. T. Ito, and the staff of the Seto Marine Biological Laboratory for use of experimental facilities, particularly to Prof. Emeritus T. Tokioka of the laboratory for advice.

\section{References}

Hagström, B.E. \& S. Lönping. 1973. The sea urchin eggs as a testing object in toxicology. Acta Pharmacol. Toxicol., 32, Suppl., 1: 1-49.

Kobayashi, N. 1971. Fertilized sea urchin eggs as a indicatory materials for marine pollution bioassay, preliminary experiment. Publ, Seto Mar. Biol. Lab., 18: 379-406.

— H. Nogami \& K. Doi. 1972. Marine Pollution Bioassay by using sea urchin eggs in the Inland Sea of Japan (Seto-Naikai). Ibid., 19: 359-381.

1974. Marine pollution bioassay by sea urchin eggs, an attempt to enhance accuracy. Ibid., $21: 377-391$.

- 1977. Preliminary experiments with sea urchin pluteus and metamorphosis in marine pollution bioassay. Ibid., 24: 9-21.

1980. Comparative sensitivity of various developmental stages of sea urchins to some chemicals. Mar. Biol., 58: 163-171.

1984. Marine ecotoxicological testing with echinoderms. In: G. Persoone, E. Jaspers \& C. Claus, eds., Ecotoxicological testing for the marine environment, Vol. 1, pp. 341-405. State Univ. Ghent and Inst. Mar. Sci. Res., Bredene.

- 1985. Marine pollution bioassay by sea urchin eggs, an attempt to enhance accuracy, II. Publ. Seto Mar. Biol. Lab., 30: 213-226. 
Pagano, G., A. Esposito, \& G.G. Giordano. 1982. Fertilization and larval development in sea urchins following exposure of gametes and embryos to cadmium. Arch. Environm. Contam. Toxicol., 11: 47-55.

, M. Cipollaro, G. Corsale, A. Esposito, A. Mineo, E. Ragucci, G.G. Giordano, N. Kobayashi, \& N. M. Trieff. 1988. Effects of sodium azide on sea urchin embryos and gametes. Teratog., Carcinog. and Mutag., 8: 363-376. 